\title{
Powering the Next Billion Devices with Wi-Fi
}

By Vamsi Talla, Bryce Kellogg, Benjamin Ransford, Saman Naderiparizi, Joshua R. Smith, and Shyamnath Gollakota

\begin{abstract}
We present the first power over Wi-Fi system that delivers power to low-power sensors and devices and works with existing Wi-Fi chipsets. We show that a ubiquitous part of wireless communication infrastructure, the Wi-Fi router, can provide far field wireless power without significantly compromising the network's communication performance. Building on our design, we prototype battery-free temperature and camera sensors that we power with $\mathrm{Wi}$-Fi at ranges of 20 and $17 \mathrm{ft}$, respectively. We also demonstrate the ability to wirelessly trickle-charge nickel-metal hydride and lithium-ion coin-cell batteries at distances of up to $28 \mathrm{ft}$. We deploy our system in six homes in a metropolitan area and show that it can successfully deliver power via Wi-Fi under real-world network conditions without significantly degrading network performance.
\end{abstract}

\section{INTRODUCTION}

In the late 19th century, Nikola Tesla dreamed of eliminating wires for both power and communication. ${ }^{16}$ As of the early 21st century, wireless communication is extremely well established-billions of people rely on it every day. Wireless power, however, has not been as successful. In recent years, near-field, short range schemes have gained traction for certain range-limited applications, like powering implanted medical device ${ }^{20}$ and recharging cars ${ }^{3}$ and phones from power delivery mats. ${ }^{8}$ More recently, researchers have demonstrated the feasibility of powering sensors and devices in the far field using RF signals from $\mathrm{TV}^{7}$ and cellular ${ }^{19}$ base stations. This is exciting, because in addition to enabling power delivery at farther distances, RF signals can simultaneously charge multiple sensors and devices because of their broadcast nature.

In this work, we show that a ubiquitous part of wireless infrastructure, the Wi-Fi router, can be used to provide far-field wireless power without significantly compromising network performance. This is attractive for three key reasons:

- Unlike TV and cellular transmissions, Wi-Fi is ubiquitous in indoor environments and operates in unlicensed spectrum (the "ISM" band) where transmissions can legally be optimized for power delivery. Repurposing Wi-Fi networks for power delivery can ease the deployment of RF-powered devices without additional power infrastructure.

- Wi-Fi uses OFDM, an efficient waveform for power delivery because of its high peak-to-average power ratio. ${ }^{17}$ Given Wi-Fi's economies of scale, Wi-Fi chipsets provide a cheap platform for sending these power-optimized waveforms, enabling efficient power delivery.
- Sensors and mobile devices are increasingly equipped with $2.4 \mathrm{GHz}$ antennas for communication via Wi-Fi, Bluetooth, or ZigBee. We can, in principle, use the same antenna for both communication and Wi-Fi power harvesting with a negligible effect on device size.

The key challenge for power delivery over Wi-Fi is the fundamental mismatch between the requirements for power delivery and the Wi-Fi protocol. To illustrate this, Figure 1 plots the voltage at a tuned harvester in the presence of Wi-Fi transmissions. While the harvester can gather energy during Wi-Fi transmissions, the energy leaks during silent periods. In this case, the Wi-Fi transmissions cannot meet the platform's minimum voltage requirement. Unfortunately for power delivery, silent periods are inherent to a distributed medium access protocol such as Wi-Fi, in which multiple devices share the same wireless medium. Continuous transmission from the router would be optimal for power delivery but would significantly degrade the performance of Wi-Fi clients and other nearby Wi-Fi networks.

This paper introduces PoWiFi, the first power over Wi-Fi system that delivers power to energy-harvesting sensors and devices while preserving network performance. We achieve this by codesigning harvesting hardware circuits and Wi-Fi router transmissions. At a high level, a router running PoWiFi imitates a continuous transmission from a harvester's perspective while minimizing the impact on Wi-Fi clients and neighboring Wi-Fi networks. The key intuition is that it is unlikely that all the Wi-Fi channels are simultaneously occupied at the same instant. PoWiFi opportunistically injects superfluous broadcast traffic (which we call power packets)

Figure 1. Key challenge with Wi-Fi power delivery. While the harvester can gather power during Wi-Fi transmissions, the power leaks during silent periods, limiting Wi-Fi's ability to meet the minimum voltage requirements of the hardware.

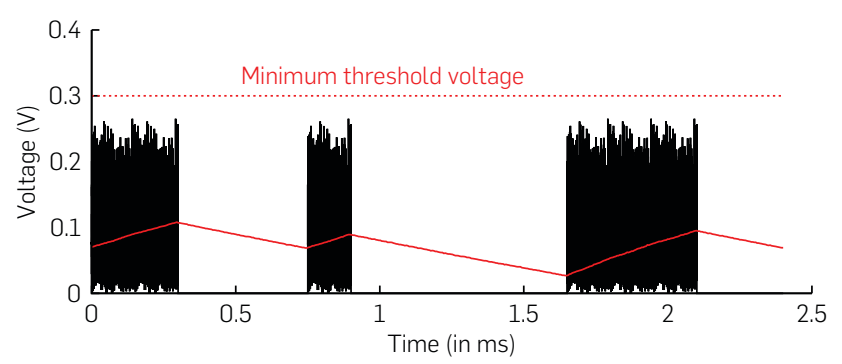

The original version of this paper was published in $A C M$ CoNext 2015. 
on nonoverlapping Wi-Fi channels to maximize the cumulative occupancy across the channels. To harvest this energy, we introduce the first multichannel harvester that efficiently harvests power across multiple Wi-Fi channels and generates the $1.8-2.4 \mathrm{~V}$ necessary to run microcontrollers and sensor systems.

To be practical, PoWiFi must not significantly degrade network performance. So our second component is a transmission mechanism that minimizes the impact on $\mathrm{Wi}-\mathrm{Fi}$ performance while effectively providing continuous power to harvesters. Specifically, to minimize the impact on associated Wi-Fi clients, PoWiFi injects power packets on a channel only when the number of data packets queued at the Wi-Fi interface is below a threshold. Further, the router transmits power packets at the highest Wi-Fi bit rates to minimize their duration, maximizing fairness to other Wi-Fi transmitters.

To further minimize its impact on neighboring Wi-Fi networks, PoWiFi uses two key techniques.

- Rectifier-aware transmissions. The key intuition is that when there are packets on the air, a harvester's temporary energy supply charges exponentially, but it also discharges exponentially during silent periods. To balance power delivery and channel occupancy, PoWiFi must minimize energy loss due to leakage. We achieve this by designing an occupancy modulation scheme that jointly optimizes the rectifier's voltage behavior and the Wi-Fi network's throughput to ensure that harvesting sensors can meet their duty-cycling requirements (see Rectifier-aware PoWiFi transmissions section).

- Scalable concurrent transmissions. A key goal is to maintain good network performance when there are multiple PoWiFi routers in an area. Our insight is that PoWiFi's power packets do not contain useful data, and so the transmissions from multiple PoWiFi routers can safely collide. Further, by making each PoWiFi router transmit random power packets, we ensure that concurrent packet transmissions do not destructively interfere to reduce available power at sensors.

We build prototype PoWiFi routers using Atheros chipsets and harvesters using off-the-shelf components. Our experiments demonstrate the following:

- The power packets at the PoWiFi router do not noticeably affect TCP or UDP throughput or webpage load times ${ }^{1}$ at an associated client. Meanwhile, PoWiFi achieves an average cumulative occupancy of $95.4 \%$ across the three nonoverlapping $2.4 \mathrm{GHz}$ Wi-Fi channels.

- PoWiFi's unintrusive transmission strategy allows neighboring Wi-Fi networks to achieve better-thanequal-share fairness, because a PoWiFi router transmits power packets at the highest bit rate to minimize its channel occupancy.

- Using a rectifier-aware transmission scheme that can adapt to a harvester's energy needs, PoWiFi's per-channel occupancy is as low as $4.4 \%$ while delivering power to a sensor $16 \mathrm{ft}$ away that reads temperature values once every minute.

- We perform a proof-of-concept evaluation of our concurrent transmission mechanism with one, three, and six PoWiFi routers. While the variance of neighboring Wi-Fi networks' throughput slightly increases, their mean throughput does not statistically differ. This shows the feasibility of scaling our design with multiple PoWiFi routers.

To demonstrate the potential of our design, we build two battery-free, Wi-Fi powered sensing systems shown in Figure 2: a temperature sensor and a camera. The devices use Wi-Fi power to run their sensors and a programmable microcontroller that collects the data and sends it over a UART interface. The camera and temperature-sensor prototypes can operate battery-free at distances of up to 17 and $20 \mathrm{ft}$, respectively, from a PoWiFi router. As expected, the duty cycle at which these sensors can operate decreases with distance. Further, the sensors can operate in through-the-wall scenarios when separated from the router by various wall materials.

We also integrate our harvester with $2.4 \mathrm{~V}$ nickel-metal hydride (NiMH) and 3.0 V lithium-ion (Li-Ion) coin-cell batteries. We build battery-recharging versions of the above sensors wherein PoWiFi trickle charges the batteries. The battery-recharging sensors can run energy-neutral operations at distances of up to $28 \mathrm{ft}$.

Finally, we deploy PoWiFi routers in six homes in a metropolitan area. Each home's occupants used the PoWiFi router for their Internet access for $24 \mathrm{~h}$. Even under realworld network conditions, PoWiFi efficiently delivers power while having a minimal impact on user experience.

Figure 2. Prototype hardware demonstrating PoWiFi's potential. The prototypes harvest energy from Wi-Fi signals through a standard 2 dBi Wi-Fi antenna (not shown). The low gain antenna ensures that the device is agnostic to the antenna orientation and placement. We developed (a) a battery free camera to capture images, (b) a temperature sensor to measure temperature, (c) a Li-ion battery charger, and (d) a NiMH battery charger.

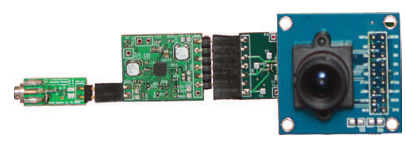

(a) Battery free camera

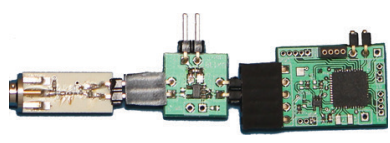

(b) Temperature sensor

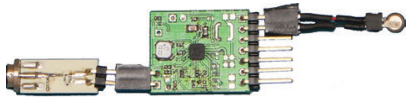

(c) Li-ion battery charger

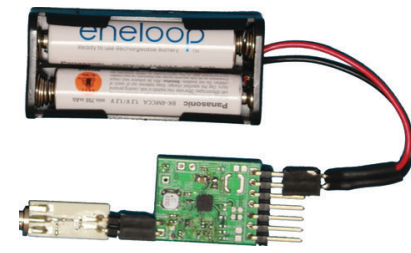

(d) NiMH battery charger 


\subsection{Limitations}

Given today's FCC $1 \mathrm{~W}$ limit on transmitters in the ISM band that Wi-Fi uses, power over Wi-Fi is limited to low-power sensors and devices and cannot, for example, recharge smartphones that require $5 \mathrm{~W}$. Further, the range of our system is determined by the sensitivity of our harvester hardware, which is built with off-the-shelf components. We believe that an ASIC design would be able to improve the harvester's sensitivity and double PoWiFi's power-delivery range. Finally, while our current design uses a single antenna, in principle we can use multiple antennas to focus more power toward a sensor and increase the range, but such optimizations are beyond the scope of this paper.

\section{UNDERSTANDING WI-FI POWER}

To understand the ability of a Wi-Fi router to deliver power, we run experiments with our organization's Asus RT-AC68U router and a temperature sensor. The router operates on Channel 5 and is set to transmit $23 \mathrm{dBm}$ power on each of its three $4.04 \mathrm{dBi}$ antennas. The temperature sensor is battery free and uses our RF harvester to draw power from Wi-Fi signals. An RF harvester is a device that converts incoming alternating current (AC) radio signals into direct current (DC). A typical RF harvester consists of two stages: a rectifier that converts the incoming radio signal oscillating at 2.4 GHz into DC voltage, and a DC-DC converter that boosts this voltage to a higher value. Every sensor or microcontroller requires a minimum voltage to run meaningful operations and the DC-DC converter ensures that these requirements are met. The key limitation in harvesting power is that every DC-DC converter has a minimum input voltage threshold below which it cannot operate. We use the DC-DC converter with the lowest threshold of $300 \mathrm{mV} .^{12}$

We place the sensor $10 \mathrm{ft}$ from the router for $24 \mathrm{~h}$ and measure the voltage at the rectifier's output throughout our experiments. We also capture the packet transmissions from the router using a high-frequency oscilloscope connected through a splitter. Over the tested period, the sensor did not reach the $300 \mathrm{mV}$ threshold. Figure 1 plots both the packet transmissions and the rectifier voltage during a period of peak network utilization. It shows that while the sensor can harvest energy during the Wi-Fi packet transmission, there is no input power during the silent slots. The energy leakages during these periods ensure that the voltage does not cross the $300 \mathrm{mV}$ threshold.

\section{PoWiFi}

PoWiFi combines two elements: (1) a Wi-Fi transmission strategy that delivers power on multiple Wi-Fi channels and (2) energy-harvesting hardware that can efficiently harvest from multiple Wi-Fi channels simultaneously. See the companion technical report ${ }^{14}$ for details on the design of the energy-harvesting hardware.

\subsection{PoWiFi router design}

Our key insight is that, at any moment, it is unlikely that all Wi-Fi channels will be occupied. Thus, PoWiFi opportunistically injects power packets across multiple Wi-Fi channels with a goal of maximizing cumulative occupancy.
Specifically, it injects 1500-byte UDP broadcast datagrams with a 100 us inter-packet delay at the highest $802.11 \mathrm{~g}$ bit rate of $54 \mathrm{Mbps}$ on the three nonoverlapping $2.4 \mathrm{GHz} \mathrm{Wi}-\mathrm{Fi}$ channels $(1,6$, and 11). A PoWiFi router enqueues these broadcast packets only when the number of frames in the wireless interface's transmit queue is below a threshold (five frames). If the queue's depth is at or above this threshold, then there are already enough power and Wi-Fi client packets in the queue to maximize channel occupancy.

PoWiFi must also provide fairness to traffic from nearby networks. Since the PoWiFi router performs carrier sensing and transmits broadcast packets at the highest $802.11 \mathrm{~g}$ bit rate, its individual frames are as short and unintrusive as possible. PoWiFi thereby provides better-than-equal-share fairness for transmissions from other networks. The rest of this section describes two techniques that further reduce PoWiFi's effect on neighboring networks.

Rectifier-aware PoWiFi transmissions. When a PoWiFi transmitter knows a harvester's electrical characteristics, it can tune its transmission strategy to precisely fit the device's power requirements. For example, suppose we need to read a temperature sensor once per minute. PoWiFi can modulate its occupancy to deliver energy to the harvester so that the sensor reaches its required voltage of $2.4 \mathrm{~V}$ just in time, minimizing the total channel occupancy subject to this goal and thereby minimizing its effect on other networks.

Empirically modeling rectifier voltage. A rectifier converts incoming Wi-Fi transmissions into DC voltage to charge a storage capacitor. Once the voltage on the capacitor reaches the required threshold $\left(V_{t h}=2.4 \mathrm{~V}\right.$ for the temperature sensor), a reading occurs. Suppose the average power at the harvester after multipath reflections and attenuation is $P_{\text {in }}$ and the channel occupancy of the PoWiFi router packets is $C$. To a first approximation, the harvester's behavior can be modeled as a DC voltage source charging a capacitor through a resistor. The difference, however, is that the approximated resistance value depends on the impedance of the harvester's diodes, which is a function of $P_{\text {in }}$ and $C$. We can write the voltage as a function of time as

$$
V(t)=V_{0} e^{-t / \tau\left(P_{i n}, C\right)}+V_{\max }\left(P_{i n}, C\right)\left(1-e^{-t / \tau\left(P_{i n}, C\right)}\right),
$$

where $V_{0}$ is the initial voltage, $\tau$ is the time constant, and $V_{\max }$ is the maximum achievable voltage. Note that both $\tau$ and $V_{\max }$ are functions of $P_{\text {in }}$ and the channel occupancy.

Given the nonlinearities of diodes, it is difficult to obtain closed-form solutions for $\tau\left(P_{i n}, C\right)$ and $V_{\text {max }}\left(P_{i n}, C\right)$. We instead connected the harvester through a cabled setup to a Wi-Fi source with variable input power and channel occupancy and measured the output voltage. We fitted the resulting data with the proposed exponential model to estimate how $\tau$ and $V_{\max }$ vary with input power and channel occupancy. The key properties of our model fitting are: (1) $V_{\max }$ is inverse-linearly proportional to the input power and channel occupancy; (2) the time constant $\tau$ is exponentially proportional to the input power and/or the channel occupancy; and (3) it takes exponentially more time for the same increment in the voltage at a higher voltage value than at a lower one. 
We next describe how PoWiFi can modulate its channel occupancy using this empirical model, while minimizing its effect on neighboring Wi-Fi networks.

Joint optimization for efficient power delivery. To reduce the impact of power packets on neighboring Wi-Fi networks, PoWiFi must minimize the total number of power packets required to collect a sensor reading. Our key intuition is that when there are packets on the air, the capacitor charges exponentially. However, when there are no packets, the voltage on the capacitor discharges exponentially. To maximize the effectiveness of power delivery, PoWiFi must minimize capacitor leakage. We achieve this by using the channeloccupancy modulation scheme described above and shown in Figure 3. In every sensor update time window $(T)$, the router transmits no power packets for a period $(T-\delta t)$, then transmits power packets for a period of $\delta t$, targeting a channel occupancy of $0<C \leq 1$. When the channel occupancy is zero, the voltage on the capacitor is very low and there is no leakage. However, when a sensor update is required, a high channel occupancy continuously charges the capacitor (minimizing leakage) to maximizes the effectiveness of power delivery. Our goal is to find $\delta t$ and $C$ to minimize the mean of the power packet occupancy given by $C * \frac{\delta t}{T}$.

We find these values by substituting different $C$ and $\delta t$ in our empirical model and computing the minimum value. We reduce the search space by noting that for a given $P_{i n}$, there is a minimum value of $C$ below which the threshold voltage is not achievable. Further, given a channel occupancy, we know the time constant that limits $\delta t$ to a maximum value of $\tau\left(P_{i n}, C\right)$. Finally, we limit the granularity by which channel occupancy can be modulated to $10 \%$. Using these values we reduce the search space to 75 points.

We note two main points. First, the above description assumes that the router can estimate the available power, $P_{i n}$, at the sensor. To bootstrap this value, PoWiFi initially transmits power packets at a high occupancy of around $90 \%$ and notes the times when the sensor outputs a reading. PoWiFi uses our empirical model to estimate $P_{\text {in }}$ for the next cycle. At the end of every cycle it re-estimates $P_{\text {in }}$ to account for wireless channel changes. Second, in the presence of multiple sensors, we can optimize the parameters to satisfy the minimum duty-cycle requirement across all the sensors, but we omit this simple extension for brevity.

Scaling with concurrent PoWiFi transmissions. A practical

\begin{abstract}
Figure 3. Rectifier-aware power Wi-Fi transmissions and corresponding rectifier voltages. The plot shows the optimized rectifier-aware PoWiFi transmission and the corresponding voltage at the storage capacitor. $\mathrm{V}_{\mathrm{th}}=2.4 \mathrm{~V}$ and $\delta \mathrm{t}=10 \mathrm{~s}$ for a temperature sensor reading every minute at the maximum operating distance.
\end{abstract}

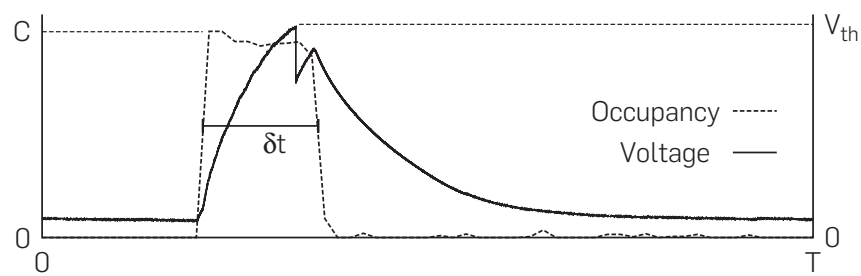

issue with each PoWiFi router independently introducing power packets is that such a system would not preserve network performance in the presence of many PoWiFi routers. Useful Wi-Fi capacity would degrade at least linearly with the number of PoWiFi routers.

To address this scaling problem, we enable concurrent transmissions from PoWiFi routers that are in decoding range of one another. Our key insight is that since power packets do not contain useful data, transmissions from multiple PoWiFi routers can safely collide. Further, if each PoWiFi router transmits a random power packet, we can ensure that concurrent packet transmissions do not destructively interfere to reduce the power available to harvesters.

Specifically, in our system, we have a leader PoWiFi router that transmits the energy pattern as shown in Figure 4 . The pattern consists of a short packet with a 1-byte payload transmitted at $54 \mathrm{Mbps}$, followed by a Distributed Interframe Space (DIFS) period and then a power packet. Other PoWiFi routers decode this short packet and join the packet transmission of the leader router within the DIFS period. This strategy ensures that all nearby PoWiFi routers transmit power packets concurrently and hence do not reduce the Wi-Fi network's capacity.

As in previous work that used concurrent transmissions, ${ }^{6}$ we enable follower routers to transmit simultaneously in software by adjusting contention-window and noise-floor parameters to prevent carrier-sense backoff, and by placing power packets in the high-priority queue. However, PoWiFi could not turnaround and begin transmission within from the software layer within a DIFS duration; we believe that with better access to the router's hardware queues, PoWiFi could turnaround within a DIFS period. Further, one can design distributed algorithms to find the leader router whose transmissions can be decoded by all other PoWiFi routers, but we consider this to be outside the scope of this paper.

\section{EVALUATION}

We build our harvester prototypes using commercial off-theshelf components on printed circuit boards. We implement PoWiFi routers using three Atheros AR9580 chipsets that independently run the algorithm in Section 3.1 on channels 1 , 6 , and 11 . The chipsets are connected via amplifiers to $6 \mathrm{dBi}$ Wi-Fi antennas separated by $6.5 \mathrm{~cm}$. Our prototype router provides Internet access to its associated clients on channel 1 via NAT and transmits at $30 \mathrm{dBm}$, the FCC limit for power in the ISM band. All our sensor and harvester benchmark evaluations were performed in a busy office network where the average cumulative occupancy across the three channels was about $90 \%$.

Both power and data packets contribute to our router's

Figure 4. Energy pattern for concurrent power packet transmissions. It consists of a short packet with a 1-byte payload transmitted at

$54 \mathrm{Mbps}$, followed by a DIFS period and a power packet transmission.

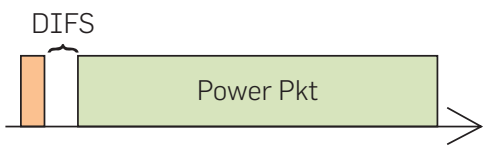


channel occupancy. To measure occupancy, we use aircrackng's airmon-ng tool to add a monitor interface to each of the router's active wireless interfaces. Then, on each monitor interface, we start tcpdump to record the radiotap headers for all frames and their retransmissions. We use tshark to extract frames sent by the router, recording the corresponding bitrate and frame size (in bytes). We then compute the average channel occupancy as $\Sigma i \in$ frames $\frac{\text { size }_{i}}{\text { rate }_{i} \times \text { total_duration }^{-}}$

\subsection{Effect on Wi-Fi clients}

PoWiFi is designed to provide high cumulative channel occupancies for power delivery while minimizing the effect on Wi-Fi traffic. To evaluate this, we deploy a PoWiFi router and evaluate its effect on Wi-Fi traffic. We use a Dell Inspiron 1525 laptop with an Atheros chipset as a client associated with our router on channel 1.

We compare four different schemes:

- Baseline. PoWiFi is disabled on the router, that is, the router introduces no extra traffic on any of its interfaces.

- BlindUDP. The router transmits UDP broadcast traffic at $1 \mathrm{Mbps}$ so as to maximize its channel occupancy.

- PoWiFi. The router sends UDP broadcast traffic at $54 \mathrm{Mbps}$ and uses the queue threshold check in Section 3.1 .

- NoQueue. The router sends UDP broadcast traffic at $54 \mathrm{Mbps}$ but disables the queue threshold check.

We evaluate PoWiFi with various Wi-Fi traffic patterns and metrics: the throughput of UDP and TCP download traffic, the page load time (PLT) of the 10 most popular websites in the United States, ${ }^{1}$ and traffic on other Wi-Fi networks in the vicinity of our benchmarking network.

Effect on UDP traffic. UDP is a common transport protocol used in media applications such as video streaming. We run iperf with UDP traffic to a client $7 \mathrm{ft}$ from the router. The client sets its Wi-Fi bitrate to $54 \mathrm{Mbps}$ and runs five sequential copies of iperf, $3 \mathrm{~s}$ apart. We repeat the experiments with target UDP data rates between 1 and $50 \mathrm{Mbps}$, and measure the achieved throughput computed over $500 \mathrm{~ms}$ intervals. All the experiments are run during a busy weekday at UW CSE, with multiple other clients and 43 other Wi-Fi networks operating at $2.4 \mathrm{GHz}$.

Figure 5a plots the average UDP throughput as a function of the 11 tested UDP data rates. The figure shows that BlindUDP significantly reduces throughput. With NoQueue, the router's kernel does not prioritize the client's iperf traffic over the power traffic. This results in roughly a halving of the iperf traffic's data rate as the wireless interface is equally shared between the two flows. With PoWiFi, however, the client's iperf traffic achieves roughly the same rate as the baseline. This result demonstrates that PoWiFi effectively prioritizes client traffic above its power traffic.

For the PoWiFi experiments above, Figure 6a plots the CDFs of individual channel occupancies on the three Wi-Fi channels. The figure shows that the individual channel occupancies are around $5-50 \%$ across the channels. The mean cumulative occupancy, on the other hand, is $97.6 \%$, demonstrating that PoWiFi can efficiently deliver power even in the presence of UDP download traffic.

Effect on TCP traffic. Next we run experiments with TCP traffic using iperf at the client. The router is configured to run the default Wi-Fi rate-adaptation algorithm. We run experiments over a period of $3 \mathrm{~h}$ with a total of 30 runs. In

Figure 5. Effect on Wi-Fi traffic. The figures show the effect of various schemes on TCP and UDP throughput as well as the page load times of the top 10 websites in the United States. ${ }^{1}$ The plots show that PoWiFi minimizes its effect on the Wi-Fi traffic. (a) UDP experiments, (b) TCP experiments, and (c) PLT experiments.

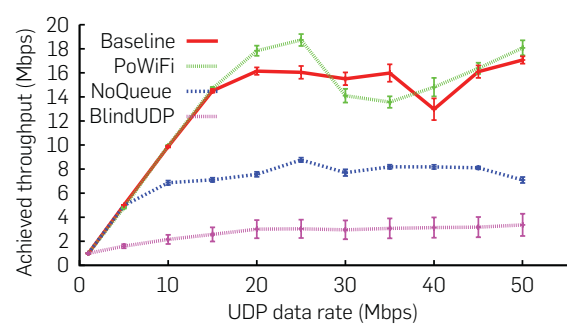

(a)

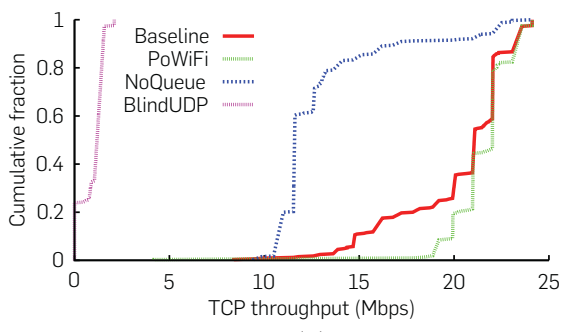

(b)

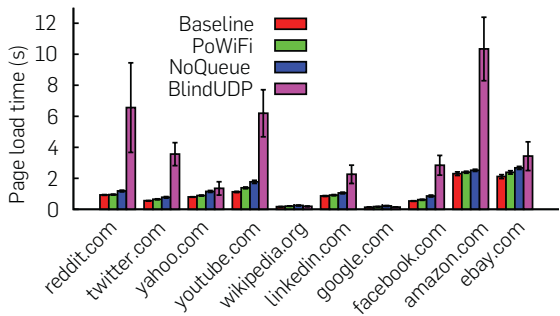

(c)

Figure 6. PoWiFi channel occupancies. The plots show the occupancies with PoWiFi for the above UDP (a), TCP (b), and PLT (c) experiments.

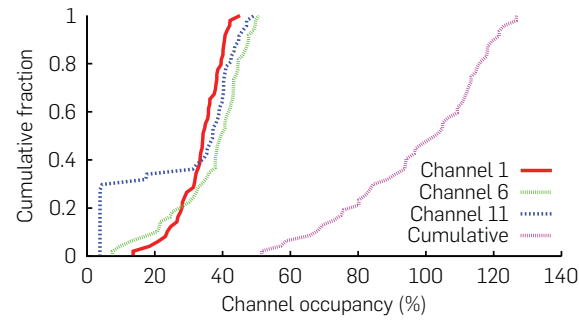

(a)

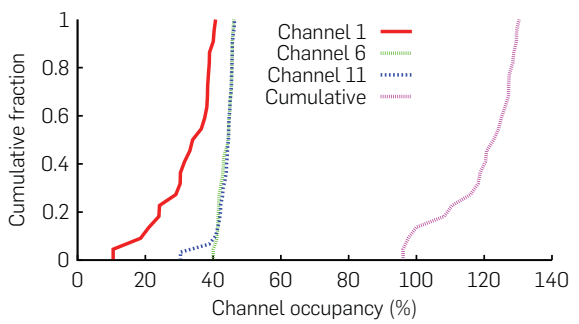

(b)

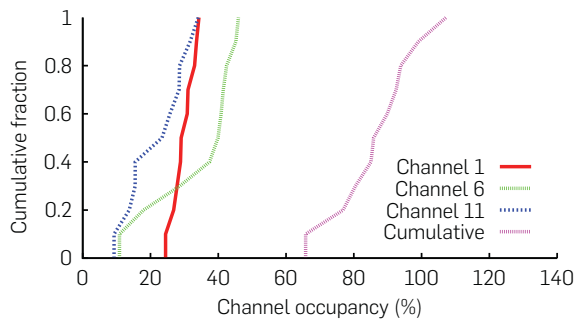

(c) 
each run, we run five sequential copies of iperf, $3 \mathrm{~s}$ apart, and compute the achievable throughput over $500 \mathrm{~ms}$ intervals, with all the schemes described above.

Figure 5b plots CDFs of the measured throughput values across all the experiments. The plot shows that BlindUDP significantly degrades TCP throughput. As before, since NoQueue does not prioritize the client traffic over the power packets, it roughly halves the achievable throughput. PoWiFi sometimes achieves higher throughput than the baseline. This is due to changes in channel conditions that occur during the 3-h experiment period. The general trend however points to the conclusion that PoWiFi does not have a noticeable effect on TCP throughput at the client.

Figure $6 \mathrm{~b}$ plots the CDFs of the channel occupancies for PoWiFi during the above experiments. The figure shows that PoWiFi has a mean cumulative occupancy of $100.9 \%$ and hence can efficiently deliver power.

Effect on PLT. We develop a test harness that uses the PhantomJS headless browser ${ }^{11}$ to download the front pages of the 10 most popular websites in the $\mathrm{US}^{1} 100 \times$ each. We clear the cache and pause for $1 \mathrm{~s}$ in between page loads. The traffic is recorded with tcpdump and analyzed offline to determine PLT and channel occupancy. The router uses the default rate adaptation to modify its Wi-Fi bit rate. The experiments were performed during a busy weekday at UW CSE over a 2-h duration.

Figure $5 c$ shows that BlindUDP significantly increases the PLT. This is expected because the $1 \mathrm{Mbps}$ power traffic occupies a much larger fraction of the medium and hence increases packet delays to clients. NoQueue improves PLT over BlindUDP, with an average delay of $294 \mathrm{~ms}$ over the baseline. PoWiFi further minimizes the delay to $101 \mathrm{~ms}$, averaged across websites. This residual delay is due to the computational overhead of PoWiFi from the per-packet checks performed by the kernel. This slows down all the processes in the OS and hence results in additional delays. However, increasing processing power and moving these checks to hardware can further reduce these delays. In our home deployments (Section 6), users did not perceive any noticeable effects on their web performance.

For completeness, we plot the CDFs of channel occupancies for PoWiFi in Figure 6c. The plot shows the same trend as before, with a mean cumulative occupancy of $87.6 \%$.

\subsection{Effect on neighboring Wi-Fi networks}

High cumulative channel occupancy transmissions. PoWiFi leverages the inherent fairness of the Wi-Fi Medium Access Control to ensure that it is fair to other Wi-Fi networks. As a worst-case evaluation, we consider a situation where PoWiFi always tries to achieve high cumulative channel occupancies at all times. To do this, we place our PoWiFi router in the vicinity of a neighboring Wi-Fi router-client pair operating on channel 1 . We configure the PoWiFi router to transmit power packets at the highest achievable channel occupancies using our algorithm on all three nonoverlapping channels. We run iperf with UDP traffic on the neighboring router-client pair at the highest data rate and measure the achievable throughput as before. We repeat the experiments for different Wi-Fi bit rates at the neighboring Wi-Fi router-client pair. We compare three schemes: BlindUDP where our router transmits UDP packets at $1 \mathrm{Mbps}$, Equalshare where we set our router to transmit the UDP packets at the same Wi-Fi bit rate as the neighboring routerclient pair, and finally PoWiFi. EqualShare provides a baseline when every router in the network gets an equal share of the wireless medium.

Figure 7a shows the throughput for the three schemes, averaged across five runs. As expected, BlindUDP significantly degrades the neighboring router-client performance. Further, this deterioration is more pronounced at the higher bit rates. With PoWiFi, however, the throughput achieved at the neighboring router-client pair is higher than EqualShare. This is because PoWiFi transmits power packets at $54 \mathrm{Mbps}$; transmissions at such high rates occupy the channel for a smaller duration than, say, a neighboring router transmitting at $16 \mathrm{Mbps}$. This property means that PoWiFi provides better than equal-share fairness to other Wi-Fi networks. We note that while our experiments are with 802.11g, PoWiFi's power packets use the highest bit rate available for $\mathrm{Wi}-\mathrm{Fi}$. Thus, the above fairness property would hold true even with 802.11n/ac.

Rectifier-aware power transmissions. Next, we evaluate the potential of our rectifier-aware technique, to significantly reduce the average channel occupancy of the power transmissions, while efficiently delivering power to the sensors. To do this, we place our battery-free temperature sen-

Figure 7. Effect of PoWiFi, rectifier aware and concurrent power transmissions on neighboring Wi-Fi networks. The plots show that PoWiFi power transmissions provide better than EqualShare throughput performance. Rectifier aware power transmissions further improve the throughput by reducing the per channel occupancy by a factor of 10. Additionally, increasing the number of concurrently transmitting PoWiFi devices does not degrade the performance of neighboring Wi-Fi devices. (a) PoWiFi bit-rates, (b) Rectifier aware throughput, (c) Rectifier aware occupancies, and (d) Concurrent transmissions.

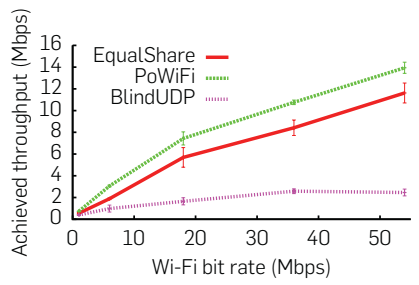

(a)

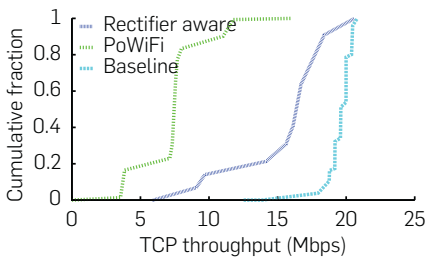

(b)

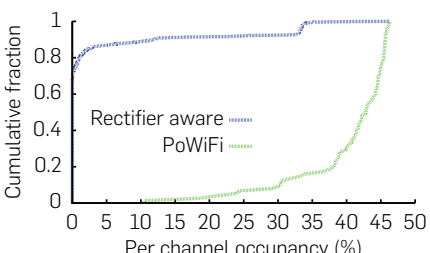

(c)

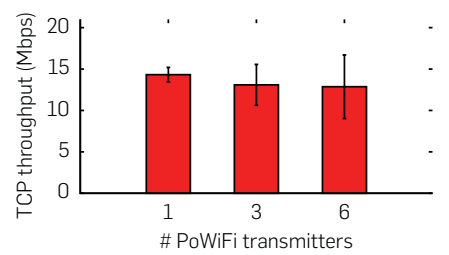

(d) 
sor close to its maximum operational range at $16 \mathrm{ft}$ from a PoWiFi router; the sensor is set to transmit a temperature value over a UART interface once every minute. The router implements the joint-optimization algorithm from "Rectifieraware PoWiFi transmissions section."

We run the experiments for a total of $10 \mathrm{~min}$ and observed that the temperature sensor achieves a mean time between updates of $59.93 \mathrm{~s}$ with a $0.43 \mathrm{~s}$ variance. More importantly, in contrast to transmitting at high channel occupancies (>90\%) all the time, our algorithm estimated that the router should transmit for a duration of $9 \mathrm{~s}$ with a $80 \%$ cumulative occupancy and stay quiet for the remaining time. Figure $7 \mathrm{~b}$ shows the throughput of an ongoing TCP flow in a neighboring Wi-Fi router-client pair, which shows that the average throughput significantly improves over high-occupancy PoWiFi and is much closer to the baseline throughput without any power packets. Figure 7c shows that rectifier aware transmissions have an average per-channel occupancy of $3.3 \%$, compared to $40 \%$ per-channel occupancy for PoWiFi transmissions - a $10 \times$ reduction in average occupancy.

Scalable concurrent power transmissions. Finally, we provide a proof-of-concept evaluation of our concurrent transmission mechanism. Wi-Fi hardware is designed to turnaround between decoding a packet and transmitting within a Short Interframe Space (SIFS) duration and hence can, in principle, easily achieve the timing requirement in Figure $4 \mathrm{~d}$. With only software access to the router, we are limited to implementing PoWiFi timing using high-speed timers and the highpriority queue. Our current software system has $36.15 \mu$ s mean turnaround time with $4.61 \mu$ s variance.

Using the above mean turnaround time as the silence period, we do a proof-of-concept evaluation. To simplify implementation, we setup a USRP N210 to transmit the pattern in Figure 4 at 30\% channel occupancy. The PoWiFi routers join this USRP transmission and concurrently transmit power packets. We evaluate the impact on the TCP throughput of a neighboring Wi-Fi router-client pair as we increase the number of PoWiFi routers. Figure $7 \mathrm{~d}$ shows that as the number of devices increases, the throughput variance slightly increases. This is because as the number of devices increases, the variance in the turnaround time between Wi-Fi power transmissions increases. The figure, however, shows that the mean throughput is only minimally affected as the number of PoWiFi devices increases from 1 to 6 . This shows the feasibility of scaling to multiple PoWiFi routers.

\section{SENSOR APPLICATIONS}

We develop Wi-Fi harvesting sensors at two ends of the energy consumption spectrum: a temperature sensor and a camera. We build both battery-free and battery-recharging versions of each.

\subsection{Wi-Fi powered temperature sensor}

We use our Wi-Fi harvester to convert incoming Wi-Fi signals into DC and power an LMT84 temperature sensor and an MSP430FR5969 microcontroller. The microcontroller reads and transmits sensor data. ${ }^{14}$ We optimize our sensor for power and each temperature measurement and transmission operation consumes only $2.77 \mu \mathrm{J}$. In the battery-recharging sensor,

we use two AAA $750 \mathrm{mAh} 2.4 \mathrm{~V}$ low discharge current NiMH battery and recharge with our battery-charging harvester (see Ref. ${ }^{14}$ for more details).

Experiments. We evaluate our temperature sensor by measuring the update rate of the sensor as function of operating distance. Specifically, we use a PoWiFi router and place both the battery-recharging and battery-free sensor at increasing distances. In the battery-free case, we measure the update rate by computing the time between successive sensor readings. In the battery-operated case, we measure the battery voltage and the charge current flowing into it from the harvester. Since, each temperature measurement and data transmission takes $2.77 \mu \mathrm{J}$, we compute the ratio of the incoming power to this value to ascertain the sensor update rate for energy-neutral operation. The average cumulative occupancy in our experiments was $91.3 \%$.

Results. Figure 8 shows that the update rate of both batteryrecharging and battery-free version of our sensors decrease with distance from the router. This is a result of less power being available and consequently less power being harvested as the distance between the router and sensor increases. Furthermore, we observe that the battery-free sensor operates upto a distance of $20 \mathrm{ft}$ whereas the battery-recharging sensor, optimized for lower input power, has better update rate at distances beyond $15 \mathrm{ft}$ and can operate up to $28 \mathrm{ft}$ from the router.

\subsection{Wi-Fi powered camera}

We use OV7670, a low-power VGA image sensor from Omnivision, interface it with an MSP430FR5969 microcontroller and power it with our harvester. We optimize our firmware for power and achieve a per-image capture energy of $10.4 \mathrm{~mJ}$. On our battery-free camera, we use an ultra-low leakage AVX BestCap $6.8 \mathrm{mF}$ super-capacitor as the storage element. Our battery-recharging camera consists of the same hardware as before, but uses our wirelessly rechargeable $1 \mathrm{mAh}$ lithium-ion coin-cell battery at $3.0 \mathrm{~V}$ (see Ref. ${ }^{14}$ for details).

Experiments 1 . We evaluate the camera by measuring the time between successive frames as a function of distance from the router. As before, we use a PoWiFi router-the observed average cumulative occupancy was $90.9 \%$ across experiments. At each distance, we wait for the camera to take at least six frames and measure the time between consecutive frames. For the battery-recharging camera, we ascertain the interframe duration for an energy-neutral image capture.

Figure 8. Sensor update rate. The temperature (camera) sensor can operate up to 20 (17) and $28(23) \mathrm{ft}$ as battery-free and energy-neutral battery-recharging, respectively. (a) Temperature Sensor (b) Camera.

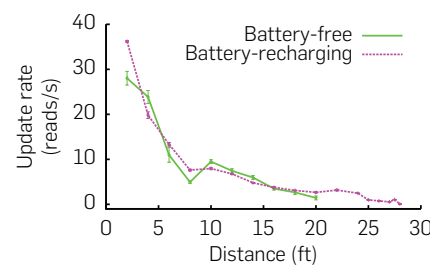

(a)

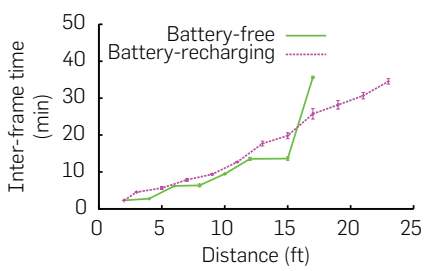

(b) 
Results 1. Figure 8b shows that the battery-free camera can operate up to $17 \mathrm{ft}$ from the router, with an image capture every $35 \mathrm{~min}$. On the other hand, the battery-recharging camera has an extended range of $23 \mathrm{ft}$ with an image capture every $34.5 \mathrm{~min}$ in an energy-neutral manner. Both the sensors have a similar image capture rate up to $15 \mathrm{ft}$ from the router. We also note that Figure $8 \mathrm{~b}$ limits the range to $23 \mathrm{ft}$ to focus on the smaller values. Our experiments, however, show that the battery-recharging camera can operate up to $26.5 \mathrm{ft}$ with an image capture every $2.6 \mathrm{~h}$.

\section{HOME DEPLOYMENT STUDY}

In Section 4.2, we showed that the channel occupancy of PoWiFi can be optimized for different sensor applications and minimize impact on neighboring Wi-Fi devices. However, PoWiFi's ability to efficiently deliver power depends on the traffic patterns of other Wi-Fi networks in the vicinity as well as the router's own client traffic, both of which can be unpredictable. So, we deploy our system in six homes in a metropolitan area and measure PoWiFi's ability to continuously achieve high channel occupancies.

Table 1 summarizes the number of users, devices, and other $2.4 \mathrm{GHz}$ routers nearby in each of our deployments. We replace the router in each home with a PoWiFi router, and the occupants use it for normal Internet access for $24 \mathrm{~h}$. Our router uses the same SSID and authentication information

Table 1. Summary of our home deployment

\begin{tabular}{|lrrrrrr|}
\hline Home \# & $\mathbf{1}$ & $\mathbf{2}$ & $\mathbf{3}$ & $\mathbf{4}$ & $\mathbf{5}$ & $\mathbf{6}$ \\
\hline Users & 2 & 1 & 3 & 2 & 1 & 3 \\
Devices & 6 & 1 & 6 & 4 & 2 & 6 \\
Neighboring APs & 17 & 4 & 10 & 15 & 24 & 16 \\
\hline
\end{tabular}

Figure 9. PoWiFi channel occupancies in home deployments. We see significant variation in per-channel occupancy values across homes. This is because PoWiFi uses carrier sense that reduces its occupancy when the neighboring networks are loaded. The cumulative occupancy, however, is high across time in all home deployments. (a) Home 1, (b) Home 2, (c) Home 3, (d) Home 4, (e) Home 5, and (f) Home 6.

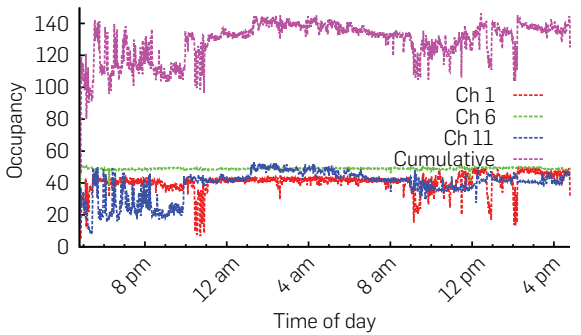

(a)

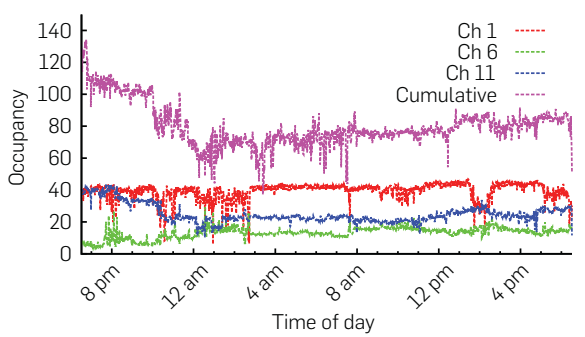

(d)

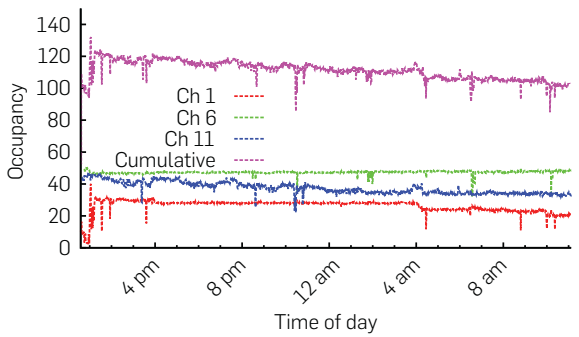

(b)

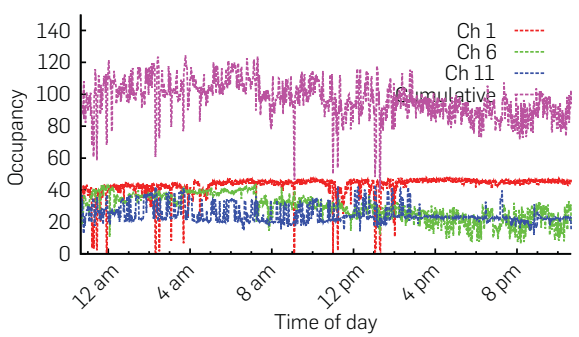

(e)

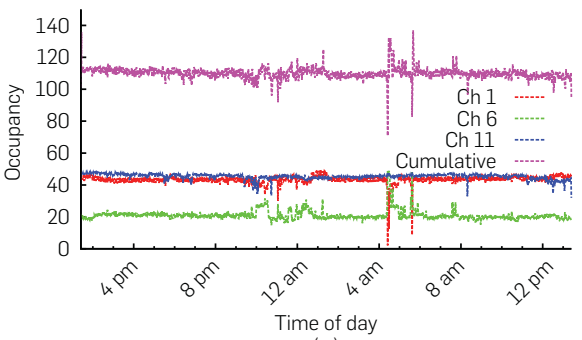

(c)

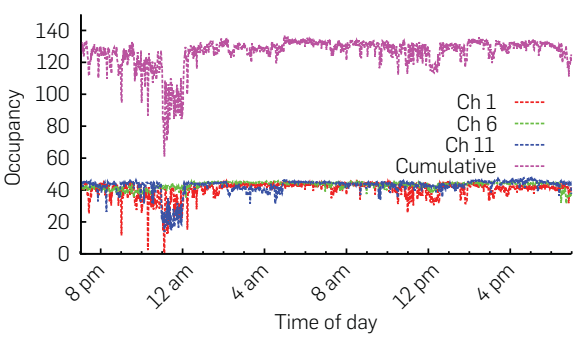

(f) 
occupancy, including both client and power traffic, dipped during this duration. This points to external causes including interference from other devices in the environment.

\section{RELATED WORK}

Early RF power delivery systems were developed as part of RFID systems to harvest small amounts of power from a dedicated $900 \mathrm{MHz}$ UHF RFID readers. ${ }^{13}$ The power harvested from RFID signals has been used to operate accelerometers, ${ }^{13}$ temperature sensors ${ }^{13}$ and recently cameras. ${ }^{9}$ Our efforts on power delivery over Wi-Fi are complimentary to RFID systems. In principle, one can combine multiple ISM bands including $900 \mathrm{MHz}, 2.4 \mathrm{GHz}$, and $5 \mathrm{GHz}$ to design an optimal power delivery system. This paper takes a significant step toward this goal.

Recently, researchers have demonstrated the feasibility of harvesting small amounts of power from ambient $\mathrm{TV}^{7}$ and cellular base station signals ${ }^{19}$ in the environment. While TV and cellular signals are stronger in outdoor environments, they are significantly attenuated indoors limiting the corresponding harvesting opportunities. The ability to power devices using Wi-Fi can augment the above capabilities and enable power harvesting indoors.

Researchers have explored the feasibility of harvesting power in the $2.4 \mathrm{GHz}$ ISM band. ${ }^{4,10,15,18}$ These efforts have demonstrated power harvesting from continuous wave (CW) transmissions and none have powered devices with existing Wi-Fi chipsets. In contrast, PoWiFi is the first power over Wi-Fi system that works with existing Wi-Fi chipsets and minimizes its impact on Wi-Fi performance. Furthermore, none of the systems power sensors and microcontrollers or recharge batteries and operate at distances demonstrated in this work.

Our work is also related to efforts from startups such as Ossia ${ }^{2}$ and Wattup. ${ }^{21}$ These claim to deliver around $1 \mathrm{~W}$ of power at $15 \mathrm{ft}$ and charge a mobile phone. ${ }^{5}$ Back-of-theenvelope calculations however show that this requires continuous transmissions with an EIRP (equivalent isotropic radiated power) of $83.3 \mathrm{dBm}(213 \mathrm{~kW})$. This not only jams the Wi-Fi channel but also is $50,000 \times$ higher power than that allowed by FCC regulations part 15 for point to multipoint links. In contrast, our system is designed to operate within the FCC limits and has minimal impact on Wi-Fi traffic. We note that in the event of an FCC exception to these startups, our multichannel design can be used to deliver such high power without having significant impact on Wi-Fi performance.

\section{CONCLUSION}

There is increasing interest in the Internet of Things where small computing sensors and mobile devices are embedded in everyday objects and environments. A key issue is how to power these devices as they become smaller and more numerous; plugging them in to provide power is inconvenient and is difficult at large scale. We introduce a novel far-field power delivery system that uses existing Wi-Fi chipsets while minimizing the impact on Wi-Fi network performance. While this is a first step toward using Wi-Fi for power delivery system, we believe that with subsequent iterations of the harvester we can significantly increase the capabilities of our system.

\section{Acknowledgments}

This research is funded in part by NSF grants CNS-1452494 and CNS-1407583, a Qualcomm Innovation Fellowship, a Intel Fellowship, and University of Washington.

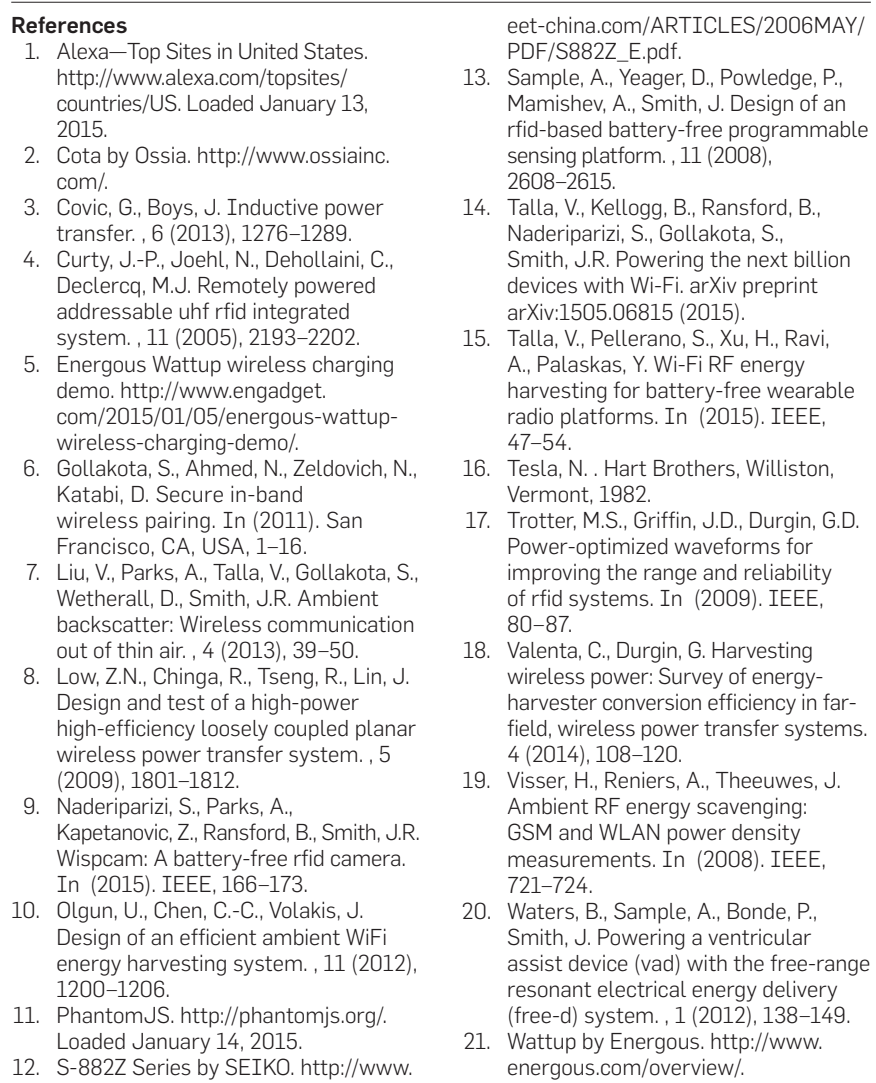

Vamsi Talla, Bryce Kellogg, Benjamin Ransford, Saman Naderiparizi, Joshua R. Smith, and Shyamnath Gollakota ( vamsit, samanp, kellogg, jrsjrs, ransford, gshyam\}@uw.edu) Department of Computer Science and Engineering University of Washington, Seattle, WA
eet-china.com/ARTICLES/2006MAY/ sensing platform. , 11 (2008)

Naderiparizi, S, Gollakota, S. Smith, J.R. Powering the next billion devices with Wi-Fi. arXiv preprin (2015). wearable Tesla

Vermont, 1982 e and reliability of rfid systems. In (2009). IEEE, arvester conversion efficiency in farfield, wireless power transfer systems. Smith, J. Powering a ventricula assist device (vad) with the free-range energous.com/overview/. 